\title{
Kooperationen mit Pflegeheimen - Lob für die Zahnärzteschaft
}

Beim Thema „(Zahn-)Ärztliche Kooperation mit Pflegeheimen“ zogen die Diskutanten eine gemischte Zwischenbilanz. Eines wurde dabei deutlich: Die Zahnärzte haben einen großen Vorsprung vor der Ärzteschaft.

„Zahnärzte möchten den Sicherstellungsauftrag für vulnerable Bevölkerungsgruppen erfüllen und Barrieren abbauen“, erklärte der Vorstandsvorsitzende der Kassenzahnärztlichen Bundesvereinigung (KZBV), Dr. Wolfgang Eßer. Die Erfolge auf diesem Gebiet sprächen für sich: Aktuell gebe es 2700 Kooperationsverträge zwischen Zahnärzten und stationären Pflegeeinrichtungen. „Jedes Heim, das zahnärztliche Versorgung wünscht, ist versorgt“, stellte Eßer heraus. Der Schlüssel zum Erfolg sei das Engagement der Zahnärzteschaft. Laut Eßer wurden bereits vor der entsprechenden Rahmenvereinbarung Leistungsanreize mit dem Gesetzgeber vereinbart und danach festgeschrieben. Zusätzlich zu den Kooperationsverträgen wies der KZBV-Chef auf das Versorgungsmodell der ambulanten aufsuchenden Versorgung hin.

Mit Blick auf den barrierefreien Aus- oder Umbau der Bestandspraxen bewertete Eßer die von der Bundesregierung im „Nationalen Aktionsplans 2.0 zur Umsetzung der Behindertenrechtskonvention der Vereinten Nationen “ zugesagte finanzielle Unterstützung positiv. In Richtung Politik machte er erneut deutlich, dass es sich dabei jedoch um Fördermittel handeln müsse und nicht um zinsgünstige Kredite.

\section{Vorbild Hessen}

Als Beispiel für eine gewinnbringende Kooperationsform verwies Manfred Mauer, Geschäftsstellenleiter des Bundesverbands privater Anbieter sozialer Dienste (bpa) Hessen, auf die Zusammenarbeit mit der dortigen Kassenzahnärztlichen Vereinigung (KZV). Beispielsweise habe die KZV Hessen gemeinsam mit dem bpa bereits vor dem Entwurf der Vereinbarung den Kontakt zu Betreibern von Pflegeheimen gesucht, um ihnen die Ängste vor Auflagen zu nehmen. Zudem gebe es laut Maurer eine intensive gemeinsame Öffentlichkeitsarbeit von KZV Hessen und bpa. In abgestimmten Rundbriefen werde wiederholt von Best-Practice-Beispielen berichtet.

Angesichts der Rekordzahl an Kooperationsverträgen zwischen Zahnärzten und stationären Pflegeheimen war Ministerialrat Joachim Becker vom Bundesministerium für Gesundheit voller Lob für die Zahnärzte. „Im Gegensatz dazu könnte man das Tempo bei den Ärzten erhöhen“, merkte Becker an. Dipl.-Med. Regina Feldmann, Hausärztin und Vorstand der Kassenärztlichen Bundesvereinigung (KBV), machte für die Ärzte fehlende Rahmenbedingungen als Problem aus. Sie sei davon überzeugt, dass es zu einer Weiterentwicklung der Kooperationsverträge komme, da die Vergütung aktuell im Bewertungsausschuss verhandelt werde. Voraussetzung für die Vorsorgung sei aber, dass es genügend Hausärzte gebe, machte Feldmann deutlich. Deshalb gelte es, das Problem des Hausärztemangels zu lösen. Große Hoffnungen setzt Feldmann dabei in den Masterplan Medizinstudium 2020.

\section{Hier steht eine Anzeige.}

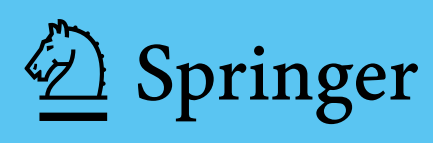

